\title{
Gibb's free energy of the spinning black holes
}

\begin{abstract}
The present gives a model for the change in Gibb's free energy of spinning black holes with corresponding change in the event horizon using the first law of black hole mechanics, mass-energy equivalence relation applied to the Gibb's free energy of the ReissnnerNordstrom black hole and concludes that the magnitude of change in free energy with corresponding change in the event horizon is approximately similar to the magnitude of change in temperature of spinning black holes with corresponding change in the mass in XRBs.

Keywords: free energy, surface gravity, XRBs
\end{abstract}

\author{
Volume 4 Issue I - 2020
}

\author{
Alok Ranjan,' Dipo Mahto² \\ 'Department of Physics, Research Scholar University, India \\ ${ }^{2}$ Associate Professor, Department of Physics, Marwari College, \\ India
}

Correspondence: Dipo Mahto, Associate Professor, Department of Physics, Marwari College, TMBU Bhagalpur, India, Tel 9006187234,Email dipomaht@hotmail.com

Received: January 31, 2020 | Published: February 12, 2020

\section{Introduction}

David Hochberg ${ }^{1}$ computed the $\mathrm{O}(\mathrm{h})$ corrections to the mass, thermal energy, entropy and free energy of the black hole due to the presence of hot conformal scalars, massless spinors and U(1) gauge quantum fields in the vicinity of the black hole using the recent solutions of the semi-classical back-reaction proble. ${ }^{1}$ You Gen Shen and Chang-Jun Gao calculated free energy and entropy of diatomic black hole due to arbitrary spin fields using the membrane model based on the brick-wall model and showed that the energy of scalar field and the entropy of Fermionic field have similar formulas containing only a numerical coefficient between them. ${ }^{2}$ David Kastor et al. ${ }^{3}$ analysed the free energy and also specific heat in the small and large black hole limits and comment upon the Hawking-Page phase transition for generic Ads-Lovelock black holes. ${ }^{3}$ Hugues Beauchesne and Ariel Edery has shown that the negative of the total Lagrangian approaches the Helmholtz free energy of a Schwarzschild black hole at the time of collapse. He also computed the numerical value of the interior Lagrangian to the expected analytical value of the interior Gibb's free energy for different initial states. ${ }^{4}$ In the present work, we have proposed a model for the change in free energy with corresponding change in the event horizon using the first law of black hole mechanics, mass-energy equivalence relation applied to the Gibb's free energy of the Reissnner-Nordstrom black hole.

\section{Theoretical discussion}

Black holes are boost machines processing the high frequency input and deliver it as low frequency output, owing to the gravitational shift and also provide a glimpse of the world at very short distance scales. This world consists of nothing, but vacuum fluctuations. ${ }^{5}$ Black hole is one of new physical phenomena predicted by General relativity ${ }^{6}$ and defined as the solution of Einstein's gravitational field equations in the absence of matter that describes the space-time around a gravitationally collapsed star. ${ }^{7}$ The gravity of a black hole is so abnormal that nothing can escape from it. The generalised form for entropy of Reissnner-Nordstrom black holes in commutative space is given by the following equation ${ }^{8}$

$$
S=\frac{A_{s}}{4}-\pi Q^{2} \ln \frac{A_{S}}{4}+\sum_{n=1}^{\infty} c_{n}\left(\frac{4}{A_{s}}\right)+C
$$

$\mathrm{c}_{\mathrm{n}}=$ quantum gravity model dependent coefficients, $\mathrm{A}_{\mathrm{s}}=$ surface area of the black holes \& $\mathrm{Q}=$ charge on the black holes.

In the case of black holes having charge $(\mathrm{Q}=0)$, then the equation (1) takes its form

$$
S=\frac{A_{s}}{4}
$$

The equation (2) is known as standard Bekenstein entropy of black hole. For the spherically symmetric and stationary or Schwarzschild black hole, its surface area is naturally given by the following equation ${ }^{9,10}$

$$
A=4 \pi R_{b h}^{2}
$$

Where the radius of event horizon for non-spinning and spinning black holes are given by equations (4) and (5) respectively.

$$
R_{b h}=\frac{2 G M}{c^{2}}
$$

And

$$
R_{b h}=\frac{G M}{c^{2}}
$$

The entropy of black holes (S) can be obtained by putting (3) into eqn (2) as:

$$
S=\pi R_{b h}^{2}
$$

The above equation is differentiated, we have

$$
d S=2 \pi R_{b h} d R_{b h}
$$

The Gibb's free energy of the Reissnner-Nordstrom black hole is given by the following equation ${ }^{3}$

$$
G=E-T S-\Phi_{H} \cdot Q
$$

Where $\mathrm{E}=\mathrm{ADM}$ mass of Reissner-Nordström black hole which gives the total energy of a space-time as defined by an observer at spatial infinity, using the Hamiltonian formalism, for an asymptotically flat space-time and The ADM mass consists of two contributions: black hole horizon and solitonic residue. It is always greater than the Schwarzschild black holes.

Where $\mathrm{C}=$ constant 
$\mathrm{T}=$ Temperature of black hole.

$\mathrm{S}=$ Entropy of black hole.

$\mathrm{Q}=$ Charge of the black hole.

$\Phi_{H}=$ Electrostatic potential at the outer horizon of black hole.

Actually the astronomical black hole is not likely to have any significant charge, because it will usually neutralised by surrounding plasma. ${ }^{11}$ Hence the charge $\mathrm{Q}=0$, the equation (8) becomes

$$
G=E-T S
$$

The product of temperature (T) and entropy $(\mathrm{S})$ for the ReissnnerNordstrom black hole is given by. ${ }^{3}$

$$
T S=\frac{1}{2} \sqrt{M^{2}-Q^{2}}
$$

For $\mathrm{Q}=0$,

$$
T S=\frac{M}{2}
$$

According to Einstein well-known mass-energy equivalence relation, we know that

$$
E=M c^{2}
$$

Putting (11) and (12) into equation (9), we have

$$
G=M c^{2}-\frac{M}{2}
$$

Putting $\mathrm{c}=1$ throughout our research work, the equation becomes

$$
\begin{gathered}
G=M-\frac{M}{2} \\
G=\frac{M}{2}
\end{gathered}
$$

The change in free energy of the Reissnner-Nordstrom black hole due to change in the mass of black hole can be obtained by differentiating the above equation

$$
d G=\frac{1}{2} d M
$$

The first law of black hole mechanics is simply an identity relating the change in mass $\mathrm{M}$, angular momentum $\mathrm{J}$, horizon area $\mathrm{A}$ and charge Q, of a black hole. The first order variations of these quantities in the vacuum satisfy. ${ }^{12,13}$

$$
\delta M=\frac{k}{8 \pi} \delta A+\Omega \delta J-v \delta Q
$$

Where $\Omega=$ Angular velocity of the horizon. $v=$ difference in the electrostatic potential between infinity and horizon.

For $\mathrm{Q}=0, \mathrm{dQ}=0$ and using the relation (2), the equation (16) becomes

$$
\delta M=\frac{\kappa}{2 \pi} \delta S+\Omega \delta J
$$

Putting (7) in the above equation, we have

$$
\delta M=\kappa R_{b h} \delta R_{b h}+\Omega \delta J
$$

For maximum spin of black hole $\left(a^{*}=1\right)$, the angular momentum of the black hole is given by. ${ }^{12}$

$$
J=M^{2}
$$

This condition corresponds to spinning black holes

Or $\delta J=2 M \delta M(20)$

Putting the above value in eqn (18), we have

$$
\begin{gathered}
\delta M=\kappa R_{b h} \delta R_{b h}+2 \Omega M \delta M \\
(1-2 \Omega M) \delta M=\kappa R_{b h} \delta R_{b h} \\
\delta M=\frac{\kappa}{(1-2 \Omega M)} R_{b h} \delta R_{b h}
\end{gathered}
$$

Putting the above value in equation (15), we have

$$
\delta G=\frac{\kappa}{2(1-2 \Omega M)} R_{b h} \delta R_{b h}
$$

The above equation gives the change in Gibb's free energy with corresponding change in the event horizon in terms of surface gravity, mass, angular velocity and event horizon of spinning black holes. In the case of spinning black holes, the surface gravity of a black hole is given by the Kerr solution. ${ }^{12,13}$

$$
\kappa=\frac{\left(M^{4}-J_{H}^{2}\right)^{1 / 2}}{2 M\left\{M^{2}+\left(M^{4}-J_{H}^{2}\right)^{1 / 2}\right\}}
$$

Each black hole is characterised by just three numbers: mass $M$, spin parameter $\mathrm{a}^{*}$ defined such that the angular momentum of the black hole is $\mathrm{a}^{*} \mathrm{GM}^{2} / \mathrm{c}^{11,12}$

Hence we have

$$
J_{H}=a * G M^{2} / c
$$

Using $\mathrm{G}=\mathrm{c}=\mathrm{h}=1$, we have

$$
J_{H}=a^{*} M^{2}
$$

The radius is smaller in the case of spinning black holes, tending to $\mathrm{GM} / \mathrm{c}^{2}$ as $\mathrm{a}^{*}$ tends to $1^{11}$ and in the case of spinning black holes having spin parameter $\left(\mathrm{a}^{*}=1\right)$, then we have,

where $J_{H}=M^{2}$

using the relation (27) into (24), we have

$$
k=0
$$

The equation (23) becomes

$$
d G=0
$$

This equation shows that the change in free energy is zero for the spinning black holes spinning at max. Spin. The above equation can be written as:

$$
\mathrm{G}=\text { constant }
$$


The above equation shows that the total Gibb's free energy of spinning black holes spinning at max. Rate has constant free energy.

Wang, D has shown that the angular velocity $\left(\Omega_{H}\right)$ evolves in a non-monotonous way in the case of thin disk-pure-accretion attaining a maximum at $\mathrm{a}^{*}=0.994$ and turns out to depend on the radial gradient of $\Omega_{p}$ near the BH horizon..$^{14}$ One black hole at the heart of galaxy NGC1365 is turning at $84 \%$ the speed light. It has reached the cosmic speed limit and cannot spin any faster without revealing its singularity. ${ }^{15}$

For convenience, let us assume $\mathrm{a}^{*}=0.9$

$J_{H}=a_{*} M^{2}$ or $J_{H}=\frac{9}{10} M^{2}$ or $J_{H}^{2}=\frac{81}{100} M^{4}$ and hence, we have

or

$$
M^{4}-J_{H}^{2}=M^{4}-\frac{81 M^{4}}{100}=\frac{19}{100} M^{4}
$$

$$
\left(M^{4}-J_{H}^{2}\right)^{1 / 2}=\frac{\sqrt{19}}{10} M^{2}=0.4358 M^{2}
$$

or $2 M\left[M^{2}+\left(M^{4}-J_{H}^{2}\right)^{1 / 2}\right]$

$$
\begin{gathered}
=2 M\left[M^{2}+0.4358 M^{2}\right] \\
=2 M\left[1.4358 M^{2}\right]==2.8716 M^{3}
\end{gathered}
$$

Putting (31) and (32) in equation (24), we have

$$
\begin{gathered}
\kappa=\frac{.4358 M^{2}}{2.8716 M^{3}} \\
\kappa=\frac{.1517}{M}
\end{gathered}
$$

Using above equation, eqn (23) becomes

$$
\begin{gathered}
\frac{d G}{d R_{b h}}=\frac{.1517 R_{b h}}{2 M(1-2 \Omega M)} \\
\frac{d G}{d R_{b h}}=\frac{.07588 R_{b h}}{M(1-2 \Omega M)}
\end{gathered}
$$

The equation (34) gives the change in Gibb's free energy with corresponding change in the event horizon in terms of mass, angular velocity and event horizon of spinning black holes.

Using $\mathrm{G}=\mathrm{h}=\mathrm{c}=1$ with equation (5), we have

$$
R_{b h}=M
$$

Using the above relation with equation (34) and solving, we have

$$
\frac{d G}{d R_{b h}}=\frac{.07588}{(1-2 \Omega M)}
$$

Some parameters of computed 2D models for progenitor stars with different masses and angular velocity of the Fe-core prior to collapse is given by ${ }^{16}$ From the data in the Table 1, it is clear that the masses of the collapsed stars are within the limit of 20 of the solar mass. We also know that the mass of the astrophysical objects like the black holes existing in XRBs ranging from $5 \mathrm{M}_{\odot}-20 \mathrm{M}_{\odot}{ }^{11}$

From the data in the Table 1, it is also clear that the maximum

\begin{tabular}{|c|c|c|c|}
\hline S. No & Mass of $B H_{s}(\mathbf{M})$ & Radius of event horizon & dG/dR $R_{b h}$ Joule \\
\hline 1 & $5 M_{\odot}$ & 7375 & $7.626 \times 10^{-33}$ \\
\hline 2 & $6 M_{\odot}$ & 8850 & $6.355 \times 10^{-33}$ \\
\hline
\end{tabular}
of the collapsed stars have zero angular velocity except one having polar angle of lateral grid boundaries. Hence we can use zero angular velocity in equation (36) and solving, we have

$$
\begin{gathered}
\frac{d G}{d R_{b h}}=0.7588 \\
d G \alpha d R_{b h}
\end{gathered}
$$

Table I Some parameters of computed 2D models for progenitor stars with different masses and angular velocity of the Fe-core prior to collapse

\begin{tabular}{llllll}
\hline S. No & Model & Mass $\left(\mathbf{M}_{\Theta}\right)$ & Angular velocity $(\Omega$ in sec-I $)$ & Polar angles $\left[\theta_{0}, \theta_{i}\right]$ & Reference \\
\hline 1 & SII.2 & 11.2 & 0 & {$[46.8,133.2]$} & 16 \\
2 & SII.2 & 11.2 & 0 & {$[46.8,133.2]$} & 16 \\
3 & SI5 & SI5 & 0 & {$[46.8,133.2]$} & 16 \\
4 & SI5P & 15 & 0 & {$[46.8,133.2]$} & 16 \\
5 & SI5r & 15 & 0.5 & {$[0,90]$} & 16 \\
6 & S20 & 20 & 0 & {$[46.8,133.2]$} & 16
\end{tabular}

Table 2 Change in free energy of spinning black holes corresponding change with event horizon in XRBs 
Table continue

\begin{tabular}{|c|c|c|c|}
\hline S. No & Mass of $B H_{s}(\mathbf{M})$ & Radius of event horizon & $\mathrm{dG} / \mathrm{d} R_{\mathrm{bh}}$ Joule \\
\hline 3 & $7 M_{\odot}$ & 10325 & $5.447 \times 10^{-33}$ \\
\hline 4 & $8 M_{\odot}$ & 11800 & $4.766 \times 10^{-33}$ \\
\hline 5 & $9 M_{\odot}$ & 13275 & $4.236 \times 10^{-33}$ \\
\hline 6 & $10 M_{\odot}$ & 14750 & $3.813 \times 10^{-33}$ \\
\hline 7 & $\| M_{\odot}$ & 16225 & $3.466 \times 10^{-33}$ \\
\hline 8 & $12 M_{\odot}$ & 17700 & $3.177 \times 10^{-33}$ \\
\hline 9 & $13 M_{\odot}$ & 19175 & $2.933 \times 10^{-33}$ \\
\hline 10 & $14 M_{\odot}$ & 20650 & $2.723 \times 10^{-33}$ \\
\hline 11 & $15 M_{\odot}$ & 22125 & $2.542 \times 10^{-33}$ \\
\hline 12 & $16 M_{\odot}$ & 23600 & $2.383 \times 10^{-33}$ \\
\hline 13 & $17 \mathrm{M}_{\odot}$ & 25075 & $2.242 \times 10^{-33}$ \\
\hline 14 & $18 M_{\odot}$ & 26550 & $2.118 \times 10^{-33}$ \\
\hline 15 & $19 M_{\odot}$ & 28025 & $2.006 \times 10^{-33}$ \\
\hline 16 & $20 M_{\odot}$ & 29500 & $1.906 \times 10^{-33}$ \\
\hline
\end{tabular}

Eqn (38) shows that the change in free energy of spinning black holes is directly proportional to the corresponding change in the radius of event horizon. This shows that the change in the free energy of spinning black holes w.r.t. the event horizon existing in XRBs remains the same except for those spinning black holes having polar angle [0, 90] degree of lateral grid boundaries. This change in the free energy of spinning black holes w.r.t. the event horizon existing in XRBs can be shown by the following graph as:

But for $\Omega=0.5 \mathrm{~s}^{-1}$, the equation (36) becomes

$$
\frac{d G}{d R_{b h}}=-\frac{0.07588}{M-1}
$$

In compared to the mass of the spinning black holes, the term 1 is negligible and hence can be neglected.

$$
\begin{gathered}
\frac{d G}{d R_{b h}}=-\frac{0.07588}{M} \\
\left|\frac{d G}{d R_{b h}}\right|=\frac{0.07588}{M}
\end{gathered}
$$

This shows the magnitude of change in free energy of spinning black hole of angular velocity 0.5 per second with about maximum spin is inversely proportional to the mass.

\section{Result and discussion}

In the present work, we have proposed a model for the change in free energy with corresponding change in the event horizon in terms of surface gravity, angular velocity, mass and radius of the event horizon of the spinning black holes given by the equation $\delta G=\frac{\kappa}{2(1-2 \Omega M)} R_{b h} \delta R_{b h}$ using the first law of black hole mechanics, mass-energy equivalence relation applied to the Gibb's free energy of the Reissnner-Nordstrom black hole. From the equation, it is clear that the surface gravity has vital role for change in free energy because its values vary for different types of black holes existing either in XRBs. In the present work, we have applied the above formula for zero surface gravity to get the change in free energy of all black holes are zero. We also have applied this work to the spinning black holes of maximum spin and obtained the change in

free energy with corresponding change in the event horizon is given by equation $\frac{d G}{d R_{b h}}=\frac{.07588 R_{b h}}{M(1-2 \Omega M)}$. After this, the work is specialised for zero angular velocity and angular velocity ( 0.5 per second) and also obtained that the change in free energy of spinning black holes is directly proportional to the corresponding change in the radius of event horizon. This shows that the change in the free energy of spinning black holes w.r.t. the event horizon existing in XRBs remains the same except for those spinning black holes having polar angle $[0,90]$ degree of lateral grid boundaries.

For the angular velocity ( $\Omega=0.5 \mathrm{sec}^{-1}$ ), we have finally obtained the magnitude of change in free energy with corresponding change in the event horizon as: $\left|\frac{d G}{d R_{b h}}\right|=\frac{0.07588}{M}$. To get the nature of variation of free energy, we have plotted the graphs between the magnitude of change in free energy with corresponding change in the event horizon in XRBs as shown in the Figure 1. 


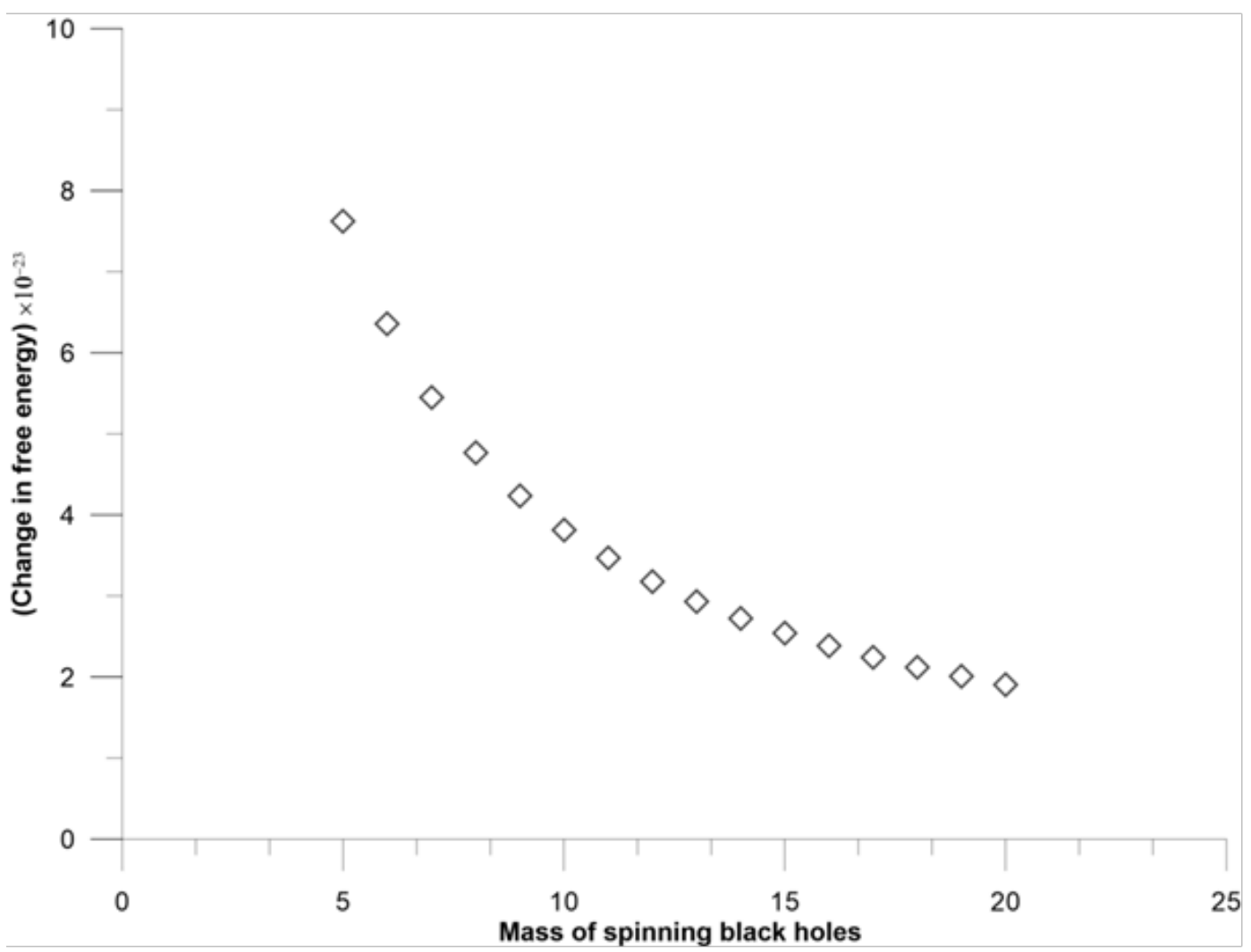

Figure I The figure shows the graph plotted between the mass of spinning black holes and their corresponding value of free energy.

From the graph plotted between the magnitude of change in free energy with corresponding change in the event horizon in XRBs, it is obvious that the magnitude of change in free energy with corresponding change in the event horizon decreases gradually with increasing the mass of the spinning black holes as the magnitude of change in temperature with corresponding change in the mass in XRBs (Ref last). It should be noted that the mass and radius of the event horizon are the same thing in the case of spinning black holes using $\mathrm{G}=\mathrm{h}=\mathrm{c}=1$ as clear from equation (39). Hence it can be concluded that the magnitude of change in free energy with corresponding change in the event horizon is approximately similar to the magnitude of change in temperature of spinning black holes with corresponding change in the mass in XRBs. ${ }^{17-19}$

\section{Conclusion}

In the present work, we can draw the following conclusions:

I. The change in Gibb's free energy with corresponding change in the event horizon depends on the surface gravity, mass, angular velocity and event horizon of spinning black holes

II. The change in Gibb's free energy of the spinning black holes of zero surface gravity is zero just like the change in entropy in the reversible process

III. The change in free energy of spinning black holes of zero angular velocity is directly proportional to the corresponding change in the radius of event horizon
IV. The Gibb's free energy of the spinning black holes of zero surface gravity is constant just like the entropy in the reversible process

V. The change in the free energy of spinning black holes w.r.t. the event horizon existing in XRBs remains the same except for those spinning black holes having polar angle $[0,90]$ degree of lateral grid boundaries

VI. The magnitude of change in free energy of spinning black hole of angular velocity 0.5 per second with about maximum spin is inversely proportional to the mass

VII. The magnitude of change in free energy with corresponding change in the event horizon is approximately similar to the magnitude of change in temperature of spinning black holes with corresponding change in the mass in XRBs.

\section{Acknowledgments}

None.

\section{Conflicts of interest}

The author declares there is no conflict of interest.

\section{References}

1. David Hochberg. Free energy and entropy for semi-classical Black Holes in the Canonical Ensemble. arXiv:gr-qc/941003401. 1994. 
2. You-Gen Shen, Chang, Jun Gao. Entropy of Diatomic Black Hole due to Arbitrary Spin Fields. Chinees Journal of Astronomy and Astrophysics. 2001;1(4):357-364.

3. David Kastor, Sourya Ray, Jennie Traschen. Mass and free energy of Lovelock Black Holes. arXiv:1106.2764v2. 2011.

4. Hugues Beauchense and Ariel Edery: Black hole free energy during charged Collapse: numerical study. arXiv:1203.2279v2. 2012.

5. Steven Corley, Ted Jacobson. Hawking spectrum and High frequency Dispersion. arXiv:[hep-th]/9601037v1. 1996.

6. Vladimir O Soloviev. Black hole statistical physics: Entropy. Institute for High energy Physics, Protvine, Russia, arXiv:[gr-qc/0507124v1. 2005. p. 54-75.

7. Dabholkar A. Black hole entropy in string theory-a window in to the quantum structure of gravity. Curr Sci. 2005;89(12):25.

8. Kourosh Nozari, Behnaz Fazlpour. Reissner-Nordstrom black hole thermodynamics in non-commutative spaces. Acta Phys Pol B. 2008;6(39):1-9.

9. Bekenstein JD. Bekenstein-Hawking Entropy, Scholarpedia. 2008;3:7375.

10. Dipo Mahto, Kanak Kumari, RK Sah, et al. Study of Non-Spinning Black Holes with Reference to the Change in Energy and Entropy. Astrophysics and Space Science. 2012;337:685-691.

11. Ramesh Narayan. Black Holes in Astrophysics. New Journal Physics 2005;7(1):1-31.
12. Dipo Mahto, Md Shams Nadeem, Mahendra Ram, et al. Gravitational Force between the Black hole and light particles in XRBs. Journal of Gravity. 2013;6:Article ID 232676.

13. Bardeen JM, Carter B, Hawking SW. The four laws of black hole mechanics. Communications in MathematicalPhysics. 1973;31(2):161170.

14. Ding-Xiong Wang. Relation between black hole spin and angular velocity of Accreting particles near the Horizon. General relativity and Gravitation. 2003;32:553.

15. Fraser Cain: How fast do black holes spin ?. Universe Today, 23 Dec. 2015.

16. Robert Buras-Schnell, Markus Rampp, HTh Janka. Improved models of stellar core collapse and still no explosion: What is missing? Physical Review Letters. 2003;90(24):241101.

17. Transchen J. An introduction to black hole evaporation. arXiv: $g r$ qc/0010055vi. 2000;1-33.

18. Wald RM. The thermodynamics of black holes. Living reviews in relativity. 2001

19. Rama Nand Mehta, Umakant Prasad, Ashok Kumar, et al. Study of variation of temperature of black holes with respect to mass in XRBs. International Journal of Astrophysics and Space Science. 2013;1(4):6163. 